\title{
Analysis of skin prick test results for 224 patients with eczema
}

\author{
Gu Hongzhi, Deng Xiangfen, Zhang Jing, Xiaoyan * \\ Department of Dermatology, The First Hospital Affiliated to AMU, Chongqing 400038, China
}

\begin{abstract}
Objective: To understand the common inhalant allergens and food allergens and their characteristics on patients with eczema in Chongqing area. Method: Skin prick tests of common inhalant allergens and food allergens were performed on 224 patients with eczema and the test results were analyzed. Results: Among the 224 patients with eczema, 137 reacted positively to one or more allergens, with a positive rate reaching $61.2 \%$; the inhalant allergens that ranked top 3 in the positive rate were dermatophagoides pteronyssinus $(41.1 \%)$, dermatophagoides farina $(37.5 \%)$, cockroach $(35.3 \%)$. The top three food allergens were shrimp (7.1\%), milk (4.5\%), egg (4\%). Conclusions: Patients with eczema were allergic to one or more allergens, and the major inhalant allergens were dermatophagoides pteronyssinus, dermatophagoides farinae and cockroaches. The positive rate of food allergens was lower that the inhalant counterparts, and the difference between male and female was not statistically significant $(\mathrm{P}>0.05)$. Allergen prick tests could facilitate identifying the inhalant allergens and food allergens of patients with eczema, improve treatment and health education of patients with eczema, and provide a reliable basis for effective prevention and normalized treatment.
\end{abstract}

\section{Introduction}

Eczema is a common dermatological disease, its pathogenetic factors are very complicated, and the specific pathogenetic cause is not completely clear. Allergens are one of the causes, so searching, reducing and removing the allergens accounts for a major solution to treat eczema. In order to identify the distribution characteristics of inhalant allergens and food allergens of patients with eczema in this region, skin prick tests of inhalant allergens and food allergens were conducted on 224 patients with eczema, and the test results are as follows.

\section{Data and methods}

2.1 Clinical data: 224 patients aged between 1 to 81 in the outpatient department from January 2019 to June 2019 were selected, wherein 91 were male, 133 were female, with an average age of 26.7 and a course of disease from 1 week to 30 years. The cases selected were patients diagnosed with eczema; all of them had not taken corticosteroids within one month before the test or antihistamines within 3 days before the test.

2.2 Test instruments: allergens prick reagents, disposable sterile prick needles

2.3 Test method: the test was performed according to the established method, as described as follows: the patients were instructed to expose the flexor side of the forearm and place the arm on the treating apparatus; the normal skin disinfected with chlorhexidine acetate and dried with a sterile swab; the allergen solution, histamine positive control solution, normal saline negative control solution was dripped onto the disinfected skin areas with a distance of 3-4 cm from each other; a disposable sterile prick needle was used to prick into the skin vertically to inject a small amount of allergens into the skin, after which the needle was uplifted and thrown away. The skin conditions were examined $30 \mathrm{~min}$ later.

2.4 Judgment standards: the ratio of the wheal area caused by the allergens and that caused by the histamine positive control solution was used to identify the level of reaction, and if no reaction occurred, the result was determined as negative; if the ratio was $1 / 3$, the reaction level was determined as $(+)$; if the ratio was $2 / 3$, the level was determined as $(++)$; if the ratio was 1 , the level was determined as $(+++)$; if the ratio exceeded 1, the level was determined as $(++++)$. The common allergens involved in the tests were inhalant allergens (dermatophagoides farinae, dermatophagoides pteronyssinus, cockroach, cladosporium herbarum bacterial genus, cross-linking and bacterial genus, candida albicans bacterial genus, pinus, mugwort, humulus scandens, small ragweed, lint, silk, dog hair, duck hair), food allergens (egg, milk, peanut, soybean, small shrimp, crab meat, carp, chicken, mutton, walnut, parsley, red pepper, mandarine, mango), the histamine positive control solution, the normal saline negative control solution, etc. 


\section{Results}

Among 224 patients with eczema, 137 reacted positively to one or more allergens (a positive rate of $61.2 \%$ ); the three top inhalant allergens that most patients were allergic to were dust mites, including dermatophagoides pteronyssinus (41.1\%), dermatophagoides farina (37.5\%), followed by cockroach $(35.3 \%)$; the top food allergens that most patients were allergic to were small shrimp (7.1\%), milk (4.5\%), egg (4\%), followed by soybean and crab meat. The inhalant allergens showed the highest positive rates (as shown in Table 1) and the difference among age groups and that between male and female was not statistically significant $(\mathrm{P}>0.05)$ (as shown in Table 2 and Table 3)

Table 1 Results of skin prick tests on 224 patients with eczema

\begin{tabular}{|c|c|c|c|c|c|}
\hline \multicolumn{3}{|c|}{ Inhalant allergens } & \multicolumn{2}{|c|}{ Food allergens } & \multirow[b]{2}{*}{ Rate (\%) } \\
\hline Allergens $\quad P$ & Positive cases & Rate (\%) & Allergens & Positive cases & \\
\hline Dermatophagoides & & & & & \\
\hline farinae & 92 & 41.1 & Small shrimp & 16 & 7.1 \\
\hline $\begin{array}{l}\text { Dermatophagoides } \\
\text { pteronyssinus }\end{array}$ & 84 & 37.5 & Milk & 10 & 4.5 \\
\hline Cockroach & 79 & 35.3 & Egg & 9 & 4 \\
\hline Mugwort & 14 & 6.2 & Soybean & 7 & 3.1 \\
\hline Alternaria alternate & 10 & 4.5 & Crab meat & 5 & 2.2 \\
\hline
\end{tabular}

Table 2. Test result comparison among age groups

\begin{tabular}{lllc}
\hline & Total number & Positive cases & Positive rate (\%) \\
\hline Aged $>50$ & 25 & 13 & 52 \\
Aged 20-49 & 114 & 77 & 67.5 \\
Aged 1-19 & 85 & 47 & 55.3 \\
\hline
\end{tabular}

Table 3. Test result comparison between male and female

\begin{tabular}{lccc}
\hline Gender & Total number & Positive cases & Rate of positive (\%) \\
Female & 133 & 87 & 65.4 \\
Male & 91 & 50 & 54.9 \\
\hline
\end{tabular}

\section{Discussion}

Eczema is a common dermatological disease and accounts for $20 \%$ or more in the amount of outpatient visits in Department of Dermatology [1]. Its main clinical symptom is pruritus, and the symptoms on the skin include erythema, papule, papulovesicle, blister, desquamation, etc. The causes and pathological mechanism are not clear, but the both internal and external factors should be considered. External factors, such as the allergens, stimuli, microorganisms, changes of ambient temperature or humidity, sunshine may cause or aggravate eczema [2].

Results of skin prick tests show that most of the patients reacted positively to one or more allergens, and patients were more susceptible to inhalant allergens than to food allergens, which was consistent with previous reports [3, 4]. The top 3 inhalant allergens were dermatophagoides pteronyssinus, dermatophagoides farinae and cockroach. This may be related to the special geographic conditions of Chongqing where the average temperature and humidity are high, and it is usually short of sunshine in winters - an environment favorable for the growth of acarid, cockroaches and mosquitos. The positive rate for food allergens was lower than that for the inhalant counterparts, lower than that reported by Zheng at al. [5]. The reason may be that Chongqing area is an inland city where shrimps and crabs account for a small proportion in the resident's diets; moreover, due to fear for eczema, some residents overreact by cutting the intake of protein-rich food like milk and eggs; the selection of limited type of food allergens (river shrimps rather than seafood) in the tests may also has some impact on the test results.

The test results show that most of the patients are allergic to 2 or more allergens, i.e. the allergic clinical diseases do not necessarily refer to allergies to one single allergen, but also a mixture of allergies to several different allergens [6]. The causes of eczema are very complicated. Some studies show that eczema is closely related to contact with allergens such as nickel sulfate, spice, rubber and p-phenylenediamine [7], and Type I and IV allergic reactions also play a part in the pathological mechanism [8]. Therefore, it is advisable that different allergen tests shall be carried out according to the actual conditions of the patients with eczema, and the possible inducing factors and pathogenic factors of the patients shall be traced. At the same time, the internal factors of the patients should be considered to conduct systematic therapies on the patients, adjust the patients' psychological and spiritual conditions to facilitate recovery. 


\section{References}

1. Zhang Jianzhong, Comprehensive Guidelines for the diagnosis and treatment of skin diseases [M]. Beijing: Chinese Medical Multimedia Press, 2014:1

2. Zhang Jianzhong. Comprehensive Guidelines for the diagnosis and treatment of skin diseases [M]. Beijing: Chinese Medical Multimedia Press, 2014:1

3. Dai Wei, Huang Xiuying, et al. Analysis of results of skin prick test for 99 patients with atopic dermatitis in Chongqing area [J]. China Journal of Leprosy and Skin Diseases, 2009, (6):477-477

4. Yao Rongfeng, Jiang Peihong, et al. The significance of allergen test on patients with eczema, allergic rhinitis and asthma[J]. Laboratory Medicine, 2015, 30(5):457-460.
5. Zheng Wen'ai, Zhong $\mathrm{Na}$ et al. Analysis of allergen test on patients with eczema and chronic urticarial [J]. China Journal of Leprosy and Skin Diseases 2013, (8):545-546.

6. Yao Rongfeng, Jiang Peihong et al. The significance of allergen test on patients with eczema, allergic rhinitis and asthma [J]. Laboratory Medicine, 2015, 30(5):457-460.

7. Huang Xiuying, Yin Rui, Zhong Baiyu et al. Analysis of results of patch test for 470 patients with dermatitis and eczema class diseases in Chongqing area [J]. Journal of Practical Dermatology, 2009, 0(4):206-207.

8. Li Mengjuan, Li Guizhenjuan. Food allergen specific antibody test for patients with eczema [J]. Journal of Practical Dermatology, 2014, 28(10):1029-1030.] 